\title{
Variación espacial y temporal de las masas de agua, nutrientes y sedimentación de la materia orgánica e inorgánica en la bahía Mejillones del sur $\left(23^{\circ} \mathrm{S}\right)$, Chile
}

\author{
Spatial and temporal variability of water masses, nutrients and sedimentation of organic \\ and inorganic matter, in Mejillones del sur bay $\left(23^{\circ} \mathrm{S}\right)$, Chile
}

\section{MAURICIO CERDA ${ }^{1, *}$, BASTIAAN KNOPPERS², JORGE VALDÉS ${ }^{3,5}$, ABDEL FETTAH SIFFEDINE $^{2,4,5}$, LUC ORTLIEB $^{4} \&$ ELISAMARA SABADINI-SANTOS ${ }^{2}$}

${ }^{1}$ Departamento de Biologia Marinha (BIOMAR) Programa de Pós-Graduação, Universidade Federal Fluminense, 24020-141 Niterói, Rio de Janeiro, Brasil

${ }^{2}$ Departamento de Geoquímica, Universidade Federal Fluminense, 24020-141 Niterói, Rio de Janeiro, Brasil

${ }^{3}$ Laboratorio de Sedimentología y Paleoambientes, Instituto de Investigaciones Oceanológicas,

Facultad de Recursos del Mar, Universidad de Antofagasta, Chile

${ }^{4}$ LOCEAN, UMR 7159 CNRS-IRD-Univ. P. \& M. Curie-MNHN, 32 Av. Henri Varagnat, 93143 Bondy, France

${ }^{5}$ Laboratorio Mixto Internacional, PALEOTRACES (Institut de Recherche pour le Developpement, Universidade Federal

Fluminense, Universidad de Antofagasta)

* Autor correspondiente: mlorenzo.mauricio@gmail.com

\begin{abstract}
RESUMEN
Durante el verano austral de 2003-2004, se estudiaron las propiedades del material particulado que sedimenta en la bahía Mejillones del Sur, y la estructura físico-química de la columna de agua. Se encontraron aguas más cálidas y oxigenadas en la superficie, una clara estratificación entre 10 y $20 \mathrm{~m}$ de profundidad y aguas más frías, densas y subóxicas bajo los $30 \mathrm{~m}$ y hasta el fondo durante el periodo de estudio. La bahía se caracterizó por la interacción de tres masas de aguas: Agua Superficial Subtropical (ASST), Agua Subantártica (ASSA), Agua Subsuperficial Ecuatorial (AESS). Se puso en evidencia que eventos moderados y cortos de surgencia con duración entre tres a ocho días alternan con períodos más prolongados de estratificación de 29 días. Durante los eventos débiles de surgencia y en el inicio del evento de estratificación prevalecieron condiciones subóxicas a microóxicas con concentraciones de nutrientes más elevados en el estrato de $10 \mathrm{~s} 10$ a $15 \mathrm{~m}$ de profundidad. La variabilidad de los niveles de saturación de oxígeno disuelto (OD) y de nutrientes con las concentraciones de nitrógeno $(\mathrm{N})$, fósforo $(\mathrm{P})$ y ácido silícico $(\mathrm{Si})$, y las razones de $\mathrm{N}: \mathrm{P}$ y Si:NID en la superficie, indicaron la presencia de una dinámica importante de los procesos de reciclaje de la materia orgánica en la columna de agua, y del aporte alóctono de nutrientes durante la surgencia. Las variaciones quincenales de los flujos totales del material particulado colectado con trampas instaladas en la bahía, sugieren que la sedimentación del material en la columna de agua se encuentra gobernada por la alternancia entre los períodos de surgencia y de estratificación. Además en las trampas de sedimentación se observó un patrón de empobrecimiento de nitrógeno en función de la profundidad, lo que sugiere condiciones de remineralización y desnitrificación en las aguas oxido-reductoras de fondo de esta bahía.
\end{abstract}

Palabras clave: bahía de Mejillones, Chile, nutrientes, sedimentación de partículas, surgencia.

\begin{abstract}
This study addresses the spatial and temporal variability of physical-chemical properties and sedimentation rates of particulate matter of the water column at a fixed station set within Bahía de Mejillones del Sur, during the austral summer of 2003-2004. The water column was generally characterized by warmer oxygen-rich surface waters, marked stratification gradients between about 10 to $20 \mathrm{~m}$ depths and by colder, denser and sub-oxic upwelling waters from $30 \mathrm{~m}$ depths to the bottom throughout studied period. The bay was governed by three different water masses: the Surface Sub-Tropical Waters (SSTW), Sub-Antarctic Waters (SAAW) and the Equatorial Sub-Surface Waters (ESSW). The surface waters exhibited a clear alternation between weak and short (three to eight days) upwelling events and longer (29 days) periods of stratification. During the weak upwelling events and at the onset of the stratification periods, sub-oxic to micro-oxic conditions prevailed with elevated nutrient concentrations at the 10 to $15 \mathrm{~m}$ depth layer. The variability of oxygen saturation levels and of the dissolved inorganic nutrients nitrogen $(\mathrm{N})$, phosphorus $(\mathrm{P})$ and silicic acid $(\mathrm{Si})$, as well as the behavior of the $\mathrm{N}: \mathrm{P}$ and Si:NID ratios, indicated the important combined effects of internal recycling of the organic matter in the surface water column and of the contribution of allochthonous nutrients from upwelled bottom waters. The fortnightly variability of the total flux of particulate material collected in sediment traps in the bay suggest that material deposition from the water column is governed by the alternation of upwelling and stratification events.
\end{abstract}


In addition, the sediment traps registered nitrogen impoverishment with increasing depths, suggesting that remineralisation processes affected the settling of particles and the denitrification of the oxydo-reducting deeper waters of the bay.

Key words: Chile, Mejillones bay, nutrients, particle sedimentation, upwelling.

\section{INTRODUCCIÓN}

La producción de partículas biogénicas en la capa superficial del océano y su remoción fraccionada en la columna de agua y sedimentos de fondo determina la distribución de los elementos biogeoquímicos en el ambiente marino (Ittekkot 1996). Este proceso es controlado por la circulación e interacción de las masas de agua y la disponibilidad de oxígeno disuelto, los cuales influyen directamente en el balance de los nutrientes y elementos trazas (Libes 1992).

El sistema de Corriente de Humboldt ( $\mathrm{SCH})$ es uno de los ecosistemas acuáticos más productivos del mundo, asociado a los vientos costeros SW y a la surgencia costera que se manifiesta con diferentes intensidades y frecuencia a lo largo de la costa de Chile (Escribano 2004). En este sistema costero, la bahía Mejillones del Sur (BM) localizada en

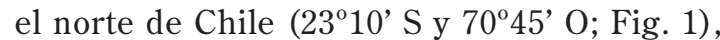
que mide 8 millas de largo y 4 millas de ancho, con una superficie aproximada de $2,500,000 \mathrm{~m}^{2}$ y un volumen de $62,500,000 \mathrm{~m}^{3}$. Presenta una configuración morfológica cóncava abierta al norte, con profundidades que alcanzan los $120 \mathrm{~m}$ en su parte externa más oceánica. La BM está delimitada al oeste por punta Angamos (PA) a lo largo de la cual las corrientes de superficie están orientadas hacia el norte. Estas corrientes junto con los vientos predominantes del suroeste contribuyen al desarrollo de un sistema de circulación interna ciclónico (Escribano 2002). La influencia de la surgencia de Agua Ecuatorial Subsuperficial (AESS), que lleva nutrientes a la capa superficial incrementa la productividad primaria de esta bahía, con tasas comparables a áreas de surgencia del Perú y África (Marín et al. 1993, González et al. 1998).

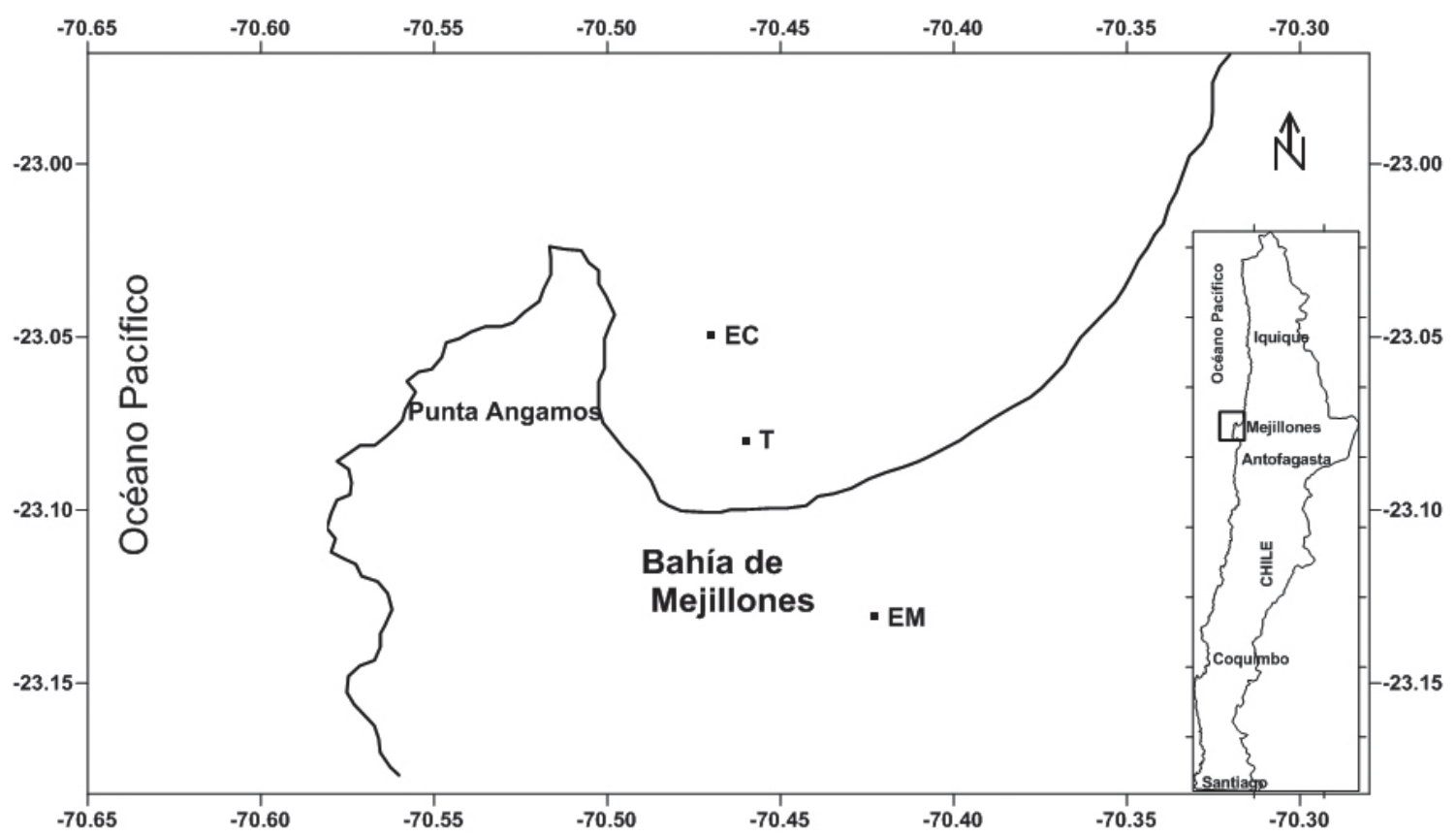

Fig. 1: Mapa de ubicación geográfica de la estación fija de muestreo (EC) y del sensor de temperatura Tidbit (T) y estación meteorológica (EM) de la bahía de Mejillones del Sur.

Geographical location of the fixed sampling station (EC), the Tidbit temperature sensor (T) and the meteorological station (EM) at bahía de Mejillones del Sur. 
En estos ambientes de alta productividad biológica el estudio de flujo de partículas con trampas de sedimentación, constituye una técnica eficiente para evaluar la composición y textura del material que sedimenta en la columna de agua, y los diferentes grados de interacción entre los procesos biológicos, físicos y geoquímicos asociados al transporte vertical y advectivos de las partículas i.e. degradación de la materia orgánica, mezcla de masas de agua, acoplamiento y desacoplamiento de la termoclina-oxiclina. (González et al. 2000, Iriarte \& González 2004, Vargas \& González 2004, Pantoja 2006, Pavés \& González 2008).

Este trabajo tiene como objetivo relacionar las condiciones hidrodinámicas, eventos de surgencia y estratificación de la columna de agua con los procesos de sedimentación de partículas, en la bahía de Mejillones durante un período estival.

\section{MÉTODOS}

Se realizó una evaluación quincenal (entre el 31 de diciembre de 2003 al 12 de marzo de 2004) de las características físico-químicas, composición de los nutrientes, materia orgánica en suspensión, pigmentos autotróficos y se determinaron tasas de sedimentación del material en la columna de agua, en una estación fija localizada en la parte central de la bahía de Mejillones $\left(23^{\circ} 05^{\prime} 13^{\prime \prime} \mathrm{S}\right.$ y $70^{\circ} 47^{\prime} 166^{\prime \prime} \mathrm{O}$ (EC), (Fig. 1)). Además se evaluó la variabilidad diurna de la temperatura superficial del mar (TSM) y el índice de pseudoesfuerzo del viento (APS) como indicadores de soporte para la estimación del impacto y variabilidad de la surgencia. El monitoreo quincenal de las condiciones oceanográficas fue realizado, mediante perfiles verticales continuos de temperatura $(\mathrm{T})$, oxígeno disuelto (OD) y salinidad (S) obtenidos con una sonda CTD Sea Bird 19 plus previamente calibrada en el laboratorio de oceanografía de la Universidad de Antofagasta. También se colectaron muestras de agua a diferentes profundidades $(1,15,30,60$ y $90 \mathrm{~m})$ con una botella Niskin (5 L), para análisis de nutrientes y composición del material en suspensión. Las muestras de agua fueron almacenadas en botellas de polietileno (1 L), previamente lavadas con una solución de $\mathrm{HCl} \mathrm{1:1}$ y agua destilada, y mantenidas en cajas de isopor con hielo $\left(4{ }^{\circ} \mathrm{C}\right)$ hasta su traslado al laboratorio. Los nutrientes inorgánicos disueltos (amonio, nitrato, nitrito, fosfato y silicato) y clorofila-a fueron analizados en el laboratorio mediante un espectrofotómetro modelo UV-1601PC Shimadzu (Strickland \& Parsons 1977, Grasshoff et al. 1983). La precisión de estos análisis, estimada mediante réplicas y su desviación estándar, fue de $3 \%$ para $\mathrm{NO}_{2}$, de $5 \%$, para $\mathrm{NO}_{3}, \mathrm{NH}_{4} \mathrm{y}$ $\mathrm{PO}_{4}$, y de $4 \%$ para $\mathrm{Si}(\mathrm{OH})_{4}$. El límite de detección $(\mu \mathrm{M})$ fue de $0.01,0.05,0.1,0.03$ y 0.1 , respectivamente.

En otra estación $\left(23^{\circ} 08^{\prime} 41^{\prime \prime}\right.$ S y $70^{\circ} 45^{\prime} 07^{\prime \prime} \mathrm{O}$, (T), Fig. 1), se instaló a $2 \mathrm{~m}$ de profundidad un sensor de temperatura de agua del tipo Titbit Onset programado con una frecuencia de muestreo de 15 minutos. Los datos de viento fueron obtenidos en la estación meteorológica que la Universidad de Chile y el Institut de Recherche pour le Developpement (IRD) mantiene en la pampa de Mejillones a $5 \mathrm{~km}$ al sur de la bahía (EM) (Fig. 1). A los datos de viento se les realizó un análisis de estadística básica, frecuencia de dirección y magnitud. Se obtuvo el ciclo diario del viento $\mathrm{y}$, a partir de este, se seleccionaron las observaciones de las $14 \mathrm{y}$ 20 horas, debido a que el viento en ese horario muestra una mayor persistencia en dirección y magnitud (Pizarro et al. 1994). Se calcularon las componentes U y V, los ejes de máxima y mínima varianza, por medio de la matriz varianza-covarianza, y el seudo esfuerzo del viento (APS), el cual representa la señal del índice de surgencia (Letelier 1998).

$\mathrm{APS}=\mathrm{V}\left(\mathrm{V}^{2}+\mathrm{U}^{2}\right)^{1 / 2}$

Donde, $\mathrm{V}$ y U son las componentes del viento en la dirección norte $(\mathrm{V})$ y oeste $(\mathrm{U})$.

La cantidad y calidad del material sedimentable en la columna de agua fue evaluada mediante dos trampas de sedimentación cilíndricas dobles de $94.9 \mathrm{~cm}^{2}$ de captación (proporción 8:1) ancladas en la estación C (EC) a profundidades de 30 y $80 \mathrm{~m}$. La trampa ubicada a $30 \mathrm{~m}$ de profundidad quedó instalada debajo de la termoclina/oxiclina para capturar el material proveniente de la capa superficial de la columna de agua asociada a la zona fótica. La trampa ubicada a 80 $\mathrm{m}$ de profundidad incorporó la zona fótica y subóxica con concentraciones de $\mathrm{OD}<0.5 \mathrm{ml} \mathrm{L}^{-1}$. Las trampas fueron instaladas a partir del día 16/01/2004, retiradas quincenalmente para la colecta del material capturado, y consecutivamente reinstaladas hasta el final de la campaña del día 12/03/2004. Las muestras obtenidas en los colectores de las trampas fueron fijadas con cloroformo $20 \%$ en solución salina concentrada para inhibir la degradación de la materia orgánica colectada (Knauer et al. 1979).

Después de cada recuperación de las trampas, el material colectado fue transferido a frascos de polietileno descontaminados y mantenidos refrigerados a $4{ }^{\circ} \mathrm{C}$ en oscuridad. En el laboratorio, los organismos nadadores fueron separado del material colectado con una malla de nylon de $500 \mu \mathrm{M}$ y el material resultante fue revisado con un estereomicroscopio para retirar copépodos de pequeño tamaño, material que no está sedimentando en la columna de agua ("swimmers"). Posteriormente, el contenido de cada colector fue homogenizado manualmente y subdividido en cuatro alícuotas. Dos de esas alícuotas fueron preservadas con formalina y lugol para realizar el análisis de plancton y pellets fecales en el microscopio óptico. Las otras dos alícuotas fueron filtradas con filtros de fibra de vidrio Whatman con una porosidad de $0.7 \mu \mathrm{M} \mathrm{GF} / \mathrm{F}$, previamente calcinados a $450^{\circ} \mathrm{C}$. En los filtros fueron determinados el peso seco del material en suspensión (Strickland \& Parsons 1977) y las concentraciones de carbono orgánico particulado total (COP) y nitrógeno orgánico particulado total (NOP) con un analizador elemental CHN Perkin Elmer 2400 (Grasshoff et al. 1983). Debido a un error en el cálculo del peso de los filtros utilizados para la colecta del material para análisis elemental, en este trabajo solamente se utilizan los contenidos de carbono y nitrógeno (en \%) y no los flujos de estos compuestos. La determinación de los pigmentos autotróficos se realizó sobre la base de la metodología propuesta por Strickland \& Parsons (1977).

El grado de relación entre los diferentes parámetros físico-químicos registrados durante el 
periodo de muestreo en la bahía de Mejillones, fue evaluado mediante un análisis de componentes principales (ACP) (Tabachnick \& Fidell 1989, James \& McCulloch 1990). La matriz con la cual se trabajo incluyó 25 muestras y 15 variables (profundidad (Z), temperatura (T), oxígeno disuelto (OD), salinidad (S), densidad (D), sílica $\mathrm{Si}(\mathrm{OH})_{4}$, fosfato $\left(\mathrm{PO}_{4}\right)$, amonio $\left(\mathrm{NH}_{4}\right)$, nitrito $\left(\mathrm{NO}_{2}\right)$, nitrato $\left(\mathrm{NO}_{3}\right)$, nitrógeno inorgánico disuelto (NID), razón $\mathrm{N}: \mathrm{P}$, razón Si:NID, material particulado en suspensión (MPS) y Clorofila-a (Clo-a). Para mejorar la detección de relaciones nolineales, todos los datos fueron transformados según Sokal \& Rohlf (1981).

\section{RESULTADOS}

\section{Columna de agua}

Para el periodo de muestreo el diagrama T-S y el triángulo de mezcla de la estación oceanográfica fija ubicada en la BM (Fig. 3) revelan la presencia de tres masas de agua: Agua Superficial Subtropical (ASST), Agua
Superficial Subantártica (ASSA) y Agua Ecuatorial Sub-superficial (AESS) (Fig. 2) (Ahumada 2002). En la superficie se observó la participación de ASST. A su vez, se pudo apreciar en el fondo de la columna de agua una mezcla de AESS y ASSA.

La distribución temporal de la temperatura y el oxígeno disuelto indica oscilaciones de la termoclina y oxiclina entre los 10 y $20 \mathrm{~m}$ de profundidad durante toda la campaña de muestreo, con excepción de los días 31/12/ 2003 y 13/2/2004 cuando se observó el ascenso de aguas frías densas y pobres en oxígeno disuelto (proceso de surgencia) (Fig. 3). Estos eventos de surgencia se caracterizaron por temperaturas superficiales (TSM) de 15 a $16{ }^{\circ} \mathrm{C}, 2$ a $4 \mathrm{ml} \mathrm{L}^{-1}$ de oxígeno disuelto y salinidades de 34.5 y 35.4. Después de estos eventos, los valores de oxígeno disuelto en la superficie aumentaron considerablemente hasta $8 \mathrm{ml} \mathrm{L}^{-1}$.

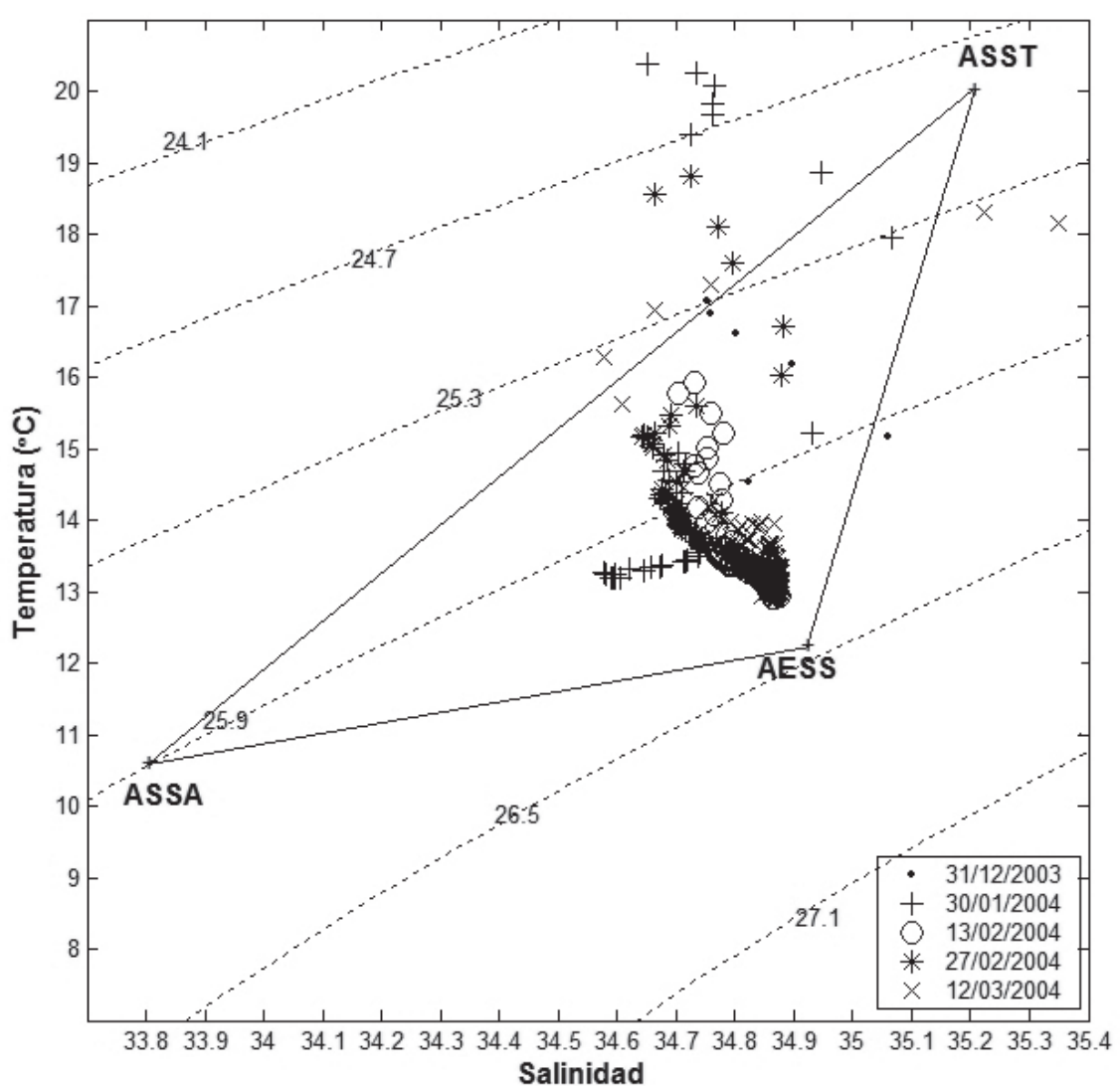

Fig. 2: Diagrama temporal T/S para todo el período de muestreo y características de las masas de agua ASST, ASSA y AESS.

$\mathrm{T} / \mathrm{S}$ diagram for the entire sampling period with the characteristic ASST, ASSA and AESS water masses. 
Índice de pseudo esfuerzo del viento y temperatura superficial del mar

Los resultados del índice de pseudo esfuerzo del viento (APS) y de la temperatura superficial del mar (TSM) se muestran en la Fig. 4. El índice APS osciló entre - 0.6 y 57.9 $\mathrm{m}^{2} \mathrm{~s}^{-2}$ con una media $14.9 \pm 11.75 \mathrm{~m}^{2} \mathrm{~s}^{-2}$. La TSM osciló entre un mínimo de $13.61^{\circ} \mathrm{C}$ y un máximo de $21.98{ }^{\circ} \mathrm{C}$ con una media de 16.45 $\pm 1.74{ }^{\circ} \mathrm{C}$. La serie temporal del índice APS y TSM mostró que en el periodo de muestreo, hubo un predominio de 38 días de aguas cálidas $\left(>15.5^{\circ} \mathrm{C}=\right.$ ASSA y ASST) y 8 días de aguas más frías $\left(<15.5{ }^{\circ} \mathrm{C}=\right.$ AESS $)$ en la superficie. Se identificó un total de 10 alteraciones cíclicas moderadas entre aguas cálidas y frías de diferentes intensidades y duración. El sistema predominante de aguas cálidas se constituyó de cinco períodos caracterizado por una temperatura media de $16.82 \pm 1.67^{\circ} \mathrm{C}$ y con valores medios bajos de APS $(13.49 \pm 12.23)$. Las pulsaciones de aguas frías fueron caracterizadas por valores medios de temperatura de $15.16 \pm 1.22{ }^{\circ} \mathrm{C}$ y valores medios más altos de APS de $18.39 \pm$ 9.66 (Fig. 4).

\section{Nutrientes, biomasa y material en suspensión}

Los perfiles verticales de nitrato $\left(\mathrm{NO}_{3}\right)$, nitrito $\left(\mathrm{NO}_{2}\right)$, fosfato $\left(\mathrm{PO}_{4}\right)$ silicato $\left(\mathrm{Si}(\mathrm{OH})_{4}\right)$, revelaron un patrón similar con concentraciones bajas en la superficie, y aumentando por debajo de la oxiclina. La mayor variabilidad temporal se encontró con los compuestos nitrogenados, principalmente nitrato y nitrito medidos en la zona media y profunda, donde predominan condiciones microxicas (< OD $\left.0.01 \mathrm{ml} \mathrm{L}^{-1}\right)$. El amonio $\left(\mathrm{NH}_{4}\right)$ presentó una distribución vertical muy homogénea, excepto el dia 30/1/2004 en el que se registró un gran aumento en la capa subsuperficial $(30 \mathrm{~m})$. Por otra parte los eventos de surgencia de los días 13/2/2004 y $12 / 3 / 2004$ se caracterizaron por concentraciones mayores de nutrientes disueltos en comparación a la media promedio del período entero, con valores de 5.97-57.14 $\mu \mathrm{M}$ de $\mathrm{NO}_{3}, 0.62-2.17 \mu \mathrm{M}$ de $\mathrm{PO}_{4}, 2.36-21.37$ $\mu \mathrm{M} \mathrm{Si}(\mathrm{OH})_{4}$ (Fig. 5).

La clorofila-a varió en la superficie entre 6 y $12 \mathrm{mg} \mathrm{L}^{-1}$ y en el fondo de 3 a $6 \mathrm{mg} \mathrm{L}^{-1}$. Por otra parte, la distribución vertical del material en suspensión fue relativamente homogénea

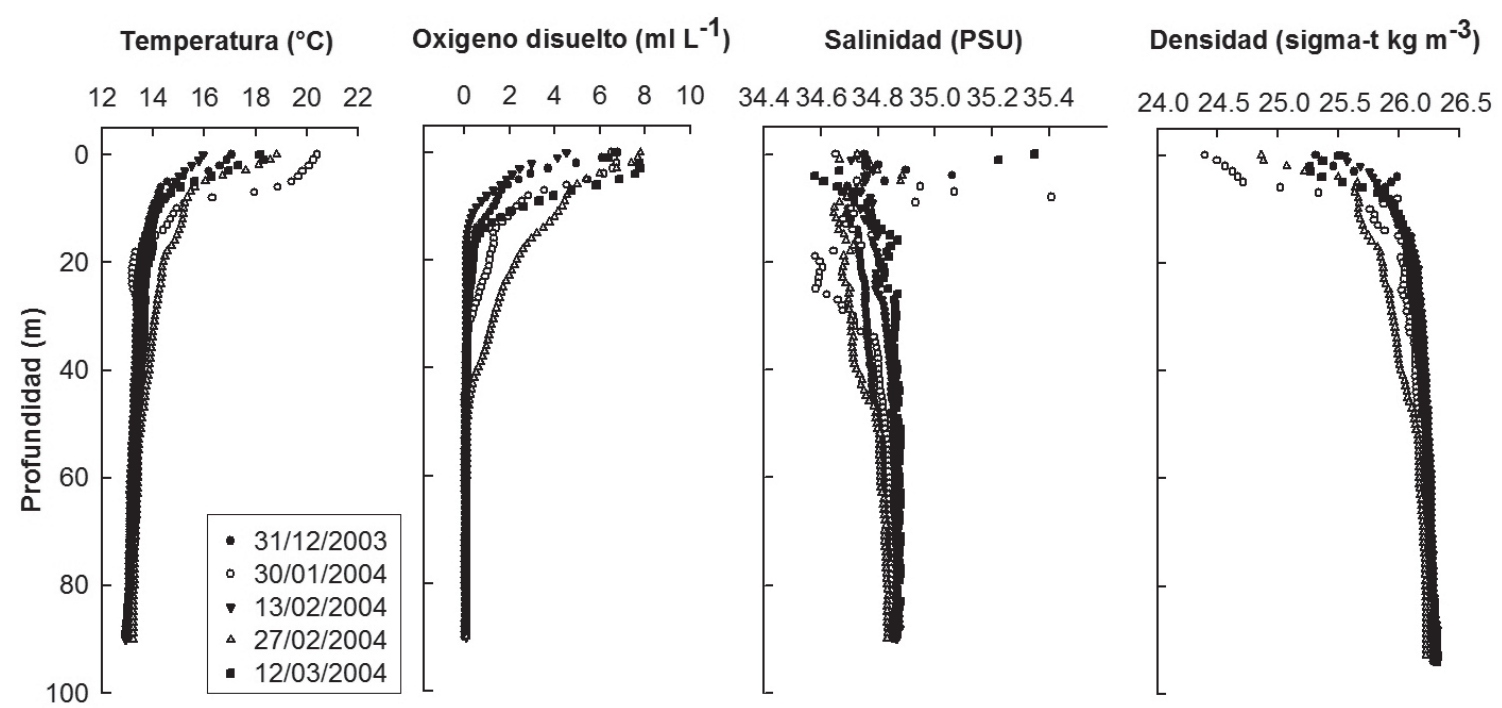

Fig. 3: Perfiles quincenales de los parámetros físico-químicos: temperatura (T), oxígeno disuelto (OD), salinidad (S) y densidad (Sigma-t), diciembre 2003 hasta marzo 2004. Nótese el intenso gradiente vertical entre la superficie y 10-20 m de profundidad.

Bi-weekly profiles of the physico-chemical parameters from December 2003 to March 2004. Temperature (T), dissolved oxygen (OD), salinity (S) and density (Sigma-t). Note the intense vertical gradients between the surface and 10 to $20 \mathrm{~m}$ depth. 




\section{Días}

Fig. 4: Serie diaria de la temperatura superficial TSM ( $2 \mathrm{~m}$ de profundidad) y el índice APS. Por debajo de la línea discontinua se destacan las pulsaciones de agua fría (surgencia) y las barras destacan los diferentes valores del índice APS, para todo el período de muestreo.

Daily series of the sea surface temperature (2 m depth) and the APS index. Below the discontinuous line, the pulsation of colder waters from upwelling can be detected from the temperature series and the bars exhibit the different intensities of the APS index during the sampling period.



$$
\mathrm{NH}_{4}{ }^{+} \mu \mathrm{M}
$$

02468101214

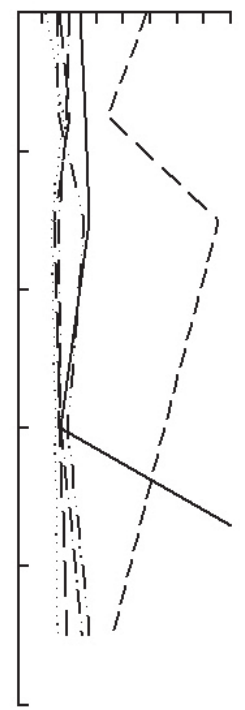

$\mathrm{PO}_{4}^{-3} \mu \mathrm{M}$

$0.00 .51 .01 .52 .02 .5 \quad 0 \quad 5 \quad 10 \quad 15 \quad 20 \quad 25$
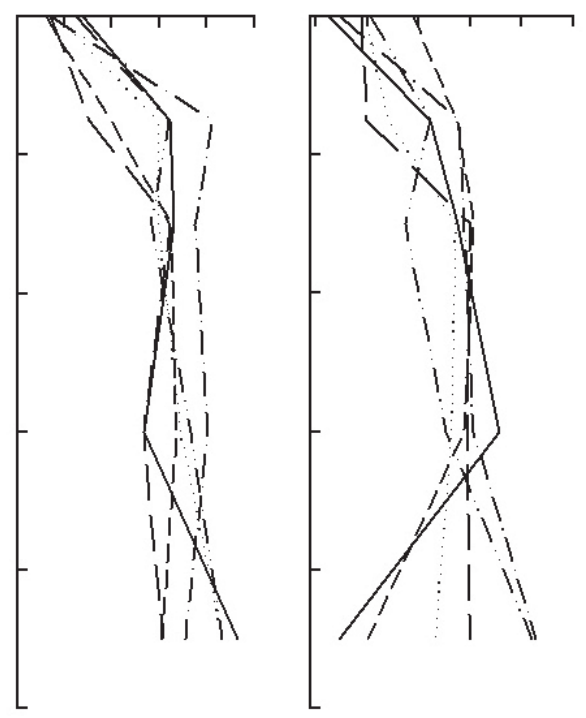

Fig. 5: Perfiles quincenales de los nutrientes disueltos, Nitrato $\left(\mathrm{NO}_{3}^{-}\right)$, Nitrito $\left(\mathrm{NO}_{2}^{-}\right)$, Amonio $\left(\mathrm{NH}_{4}^{+}\right)$, Fosfato $\left(\mathrm{PO}_{4}^{-3}\right)$ y Acido silícico $\left(\mathrm{Si}(\mathrm{OH})_{4}\right)$.

Bi-weekly profiles of dissolved nutrients, Nitrate $\left(\mathrm{NO}_{3}^{-}\right)$, Nitrite $\left(\mathrm{NO}_{2}{ }^{-}\right)$, Ammonia $\left(\mathrm{NH}_{4}^{+}\right)$, Phosphate $\left(\mathrm{PO}_{4}^{-3}\right)$ and Silicic acid $\left(\mathrm{Si}(\mathrm{OH})_{4}{ }^{-}\right)$. 
en comparación con la distribución de los otros parámetros. En tanto, la variabilidad temporal de la clorofila-a, tanto en la superficie como en el fondo, tuvo un patrón claro, i.e. los periodos sin surgencia se caracterizaron por concentraciones más elevadas de 5 a $9 \mathrm{mg} \mathrm{L}^{-1}$ en comparación con el evento de surgencia del día 13/02/2004 con 2 a 5 mg L-1.

\section{Sedimentación de partículas}

E1 material colectado por las trampas de sedimentación, después de la remoción del zooplancton (principalmente, copépodos calanoideos), se caracterizó por organismos fitoplanctónicos intactos y detritos inmersos en una matriz de agregados amorfos, pellets fecales de formas elipsoidales y alargadas y materia orgánica floculante. Las cadenas de diatomeas del genero Skeletonema y especies céntricas, tales como Thalassiosira sp., fueron ejemplos frecuentes del fitoplancton (fitodetritos) encontrados.
Los flujos del material particulado y equivalentes de clorofila sedimentaria presentaron diferencias en el tiempo, destacándose el periodo del 13 al 27/2/2004 con mayores valores en ambas profundidades $(30-80 \mathrm{~m})$. Los mayores porcentajes de carbono orgánico particulado (COP) y nitrógeno orgánico particulado (NOP) fueron encontrados en las trampas de sedimentación de $30 \mathrm{~m}$. Se encontró una disminución de COP y NOP de alrededor de $70 \%$ en las trampas de sedimentación de $80 \mathrm{~m}$ respecto de las de 30 m, durante el período del 16 al 30/1/2004 (Tabla 1).

\section{DISCUSIÓN}

Las condiciones oceanográficas en la bahía de Mejillones son complejas debido a la interacción entre las diversas corrientes y masas de agua de áreas adyacentes. La propia configuración geomorfología de la bahía

TABLA 1

Tasas de sedimentación de SST, equivalencia en clorofila-a, y porcentajes totales de COP, NOP, y la razón $\mathrm{C}: \mathrm{N}$ del material, en trampas cilíndricas a 30 y $80 \mathrm{~m}$ de profundidad.

Sedimentation rates, total suspend solids (TSS), chlorophyll-a, percentage of particulate organic carbon (POC), particulate organic nitrogen $(\mathrm{PON})$ and the C:N molar ratio of the sediment traps at 30 and $80 \mathrm{~m}$ of depth.

\begin{tabular}{|c|c|c|c|c|}
\hline & $\begin{array}{l}\text { Periodo de exposición } \\
\text { Parámetro }\end{array}$ & $\begin{array}{l}\text { Periodo de exposición } \\
\text { Parámetro }\end{array}$ & $\begin{array}{c}\text { Periodo de exposición } \\
\text { Parámetro }\end{array}$ & $\begin{array}{l}\text { Periodo de exposición } \\
\text { Parámetro }\end{array}$ \\
\hline Trampa de Sedimentación & $\begin{array}{l}\text { 16/01/2004-30/01/2004 } \\
\text { Flujo vertical }\left(\mathrm{g} \mathrm{m}^{2} \mathrm{~d}^{-1}\right)\end{array}$ & $\begin{array}{c}\text { 30/01/2004-13/02/2004 } \\
\text { Flujo vertical }\left(\mathrm{g} \mathrm{m}^{2} \mathrm{~d}^{-1}\right)\end{array}$ & $\begin{array}{l}\text { 13/02/2004-27/02/2004 } \\
\text { Flujo vertical }\left(\mathrm{g} \mathrm{m}^{2} \mathrm{~d}^{-1}\right)\end{array}$ & $\begin{array}{c}27 / 02 / 2004-12 / 03 / 2004 \\
\text { Flujo vertical }\left(\mathrm{g} \mathrm{m}^{2} \mathrm{~d}^{-1}\right)\end{array}$ \\
\hline \multicolumn{5}{|l|}{ Profundidad (m) } \\
\hline Trampa 30 & 4.75 & 2.50 & 6.26 & Sin datos \\
\hline \multirow[t]{2}{*}{ Trampa 80} & 1.57 & 1.19 & 1.67 & 21.86 \\
\hline & $\begin{array}{l}\text { Flujo vertical equivalente } \\
\text { Clorofila }\left(\mathrm{g} \mathrm{m}^{2} \mathrm{~d}^{-1}\right)\end{array}$ & $\begin{array}{l}\text { Flujo vertical equivalente } \\
\text { Clorofila }\left(\mathrm{g} \mathrm{m}^{2} \mathrm{~d}^{-1}\right)\end{array}$ & $\begin{array}{l}\text { Flujo vertical equivalente } \\
\text { Clorofila }\left(\mathrm{g} \mathrm{m}^{2} \mathrm{~d}^{-1}\right)\end{array}$ & $\begin{array}{l}\text { Flujo vertical equivalente } \\
\text { Clorofila }\left(\mathrm{g} \mathrm{m}^{2} \mathrm{~d}^{-1}\right)\end{array}$ \\
\hline Trampa 30 & 0.58 & 0.14 & 0.22 & Sin datos \\
\hline \multirow[t]{2}{*}{ Trampa 80} & 0.11 & 0.11 & 0.15 & 0.44 \\
\hline & COP $(\%)$ & COP $(\%)$ & COP $(\%)$ & COP $(\%)$ \\
\hline Trampa 30 & 4.20 & 2.80 & 3.93 & Sin datos \\
\hline \multirow[t]{2}{*}{ Trampa 80} & 1.01 & 1.44 & 2.01 & 4.87 \\
\hline & NOP (\%) & NOP (\%) & NOP $(\%)$ & NOP $(\%)$ \\
\hline Trampa 30 & 0.51 & 0.27 & 0.54 & Sin datos \\
\hline \multirow[t]{2}{*}{ Trampa 80} & 0.10 & 0.17 & 0.20 & 0.48 \\
\hline & Razón C:N & Razón C:N & Razón C:N & Razón C:N \\
\hline Trampa 30 & 8.24 & 8.47 & 7.28 & Sin datos \\
\hline Trampa 80 & 10.10 & 10.77 & 10.05 & 11.69 \\
\hline
\end{tabular}


caracterizada por un grado de enclaustramiento, propicia una retención de las masas de agua en el sector sur, condición denominada como "sombra de surgencia" (Marín et al. 2003). Durante la ausencia del proceso de surgencia, la masa de agua identificada en la superficie es ASST separada por una fuerte picnoclina de AESS y ASSA. Una leve discrepancia es generada por los diagramas T/S lo cual indica que el ASSA interacciona hasta un cierto grado con las demás masas de agua, aun cuando el impacto de AESS es predominante en la BM (Fig. 2).

La pulsación de la surgencia y su impacto sobre la bahía de Mejillones dependen de la variabilidad estacional del viento $\mathrm{SW}$, más intenso durante primavera-verano y más débil en el otoño-invierno, y de la variabilidad estacional de la intensidad y proximidad a la costa de la corriente subsuperficial Perú-Chile (Cañón \& Morales 1985). El patrón de circulación dentro de la bahía es ciclónico y directamente influenciado por el foco principal de surgencia de Punta Angamos (Escribano 2002). Los pulsos de surgencia identificados durante el período de estudio estuvieron asociados a vientos $\mathrm{SW}$ intensos y valores más altos del índice APS, dentro del rango intermedio de 20-40 $\mathrm{m}^{2} \mathrm{~s}^{-2}$, lo que según Rutllant (1993) ${ }^{1}$ ya es propicio para la generación de surgencia, dependiendo de la duración del viento. Los resultados de la TSM revelaron un evento de surgencia intensa de ocho días de duración y cuatro pulsos más cortos y menos fuertes con duración de 1 a 3 días, lo que concuerda con lo reportado por Marín et al. (1993) y Escribano et al. (2002) quienes detectaron en esta bahía pulsos de surgencia con duración de 4-5 días y 1-3 días, respectivamente.

Patrones de variación vertical y temporal de los parámetros físico-químicos en la columna de agua

El análisis de componentes principales mostró que el primer componente (PC1) explica el

$\overline{1}$ RUTLLANT J (1993) Coastal lows and associated southerly wind events in North-Central Chile. Preprints Fourth International Conference on Meteorology and Oceanography of the Southern Hemisphere. Hobart, Australia, 29 March -.2 April 1993. American Meteorological Society, p. 268-269.
$52.1 \%$ de la varianza total, y el segundo componente (PC2) explica el $17.4 \%$ de la varianza total. El principal gradiente relacionado con PC1 (Fig 6.) muestra en la columna de agua una clara separación entre la superficie $(0-15 \mathrm{~m})$ ligado positivamente con las muestras $1,2,3,4$ y 5 relacionadas con aguas cálidas y oxigenadas y aguas de fondo que se proyectan negativamente en oposición ligadas a las muestras 20, 23, 24 y 25 relacionadas con aguas más densas y ricas en nutrientes disueltos $\left(\mathrm{NO}_{3}, \mathrm{NO}_{2}, \mathrm{PO}_{4} \mathrm{y}\right.$ $\left.\mathrm{Si}(\mathrm{OH})_{4}\right)$. Este gradiente fue corroborado por el peso de las variables en los ejes (Fig. 6). Esta primera componente principal sintetiza el efecto preponderante de las condiciones hidrológicas sobre la bahía de Mejillones, las que se caracterizan por la predominancia en el fondo de aguas de surgencia (AESS) ricas en nutrientes y aguas temperadas cálidas (ASST), lo que concuerda con lo reportado por Marín \& Olivares (1999). El segundo eje, formado por las contribuciones positivas de MPS, $\mathrm{NH}_{4}$, $\mathrm{N}: \mathrm{P}$ y Clo-a, asociado a muestras superficiales $\mathrm{y}$ aguas intermedias, revelan un ambiente rico en material particulado en suspensión, en amonio orgánico que favorece al crecimiento de la biomasa fitoplanctónica con una relación ideal para la demanda del fitoplancton de N:P 16:1 (Redfield 1963). Por otra parte, las coordenadas negativas se encuentran ligadas a la razón Si:NID con valores de $2: 1$ en la superficie y $3: 1$ en el fondo, revelando un desequilibrio en la relación para la demanda ideal fitoplanctónica que es de 1:1. De esta manera, sugerimos que el segundo componente sintetiza las condiciones de un reciclaje de la materia orgánica en aguas superficiales e intermedias asociado a una fuerte estratificación durante las condiciones de verano en la bahía de Mejillones.

La sedimentación y su efecto sobre la materia orgánica y biomasa fitoplanctónica de la columna de agua

El proceso de sedimentación del material particulado en suspensión puede ser considerado como un mecanismo de extracción del material en exceso ("ballast material”) de la capa superficial de agua. Este mecanismo evita la acumulación de material refractario y promueve la manutención de 
elementos esenciales en la superficie en proporciones adecuadas para la sustentación de la productividad primaria (Heussner et al. 1987). La mayor parte del material particulado que sedimenta a través de la columna de agua de ambientes oceánicos tiene su origen en las capas superficiales del océano. Los flujos verticales de la fracción orgánica equivalen a las tasas de producción primaria nueva, sustentada por el aporte alóctono de nutrientes, que puede alcanzar hasta el $50 \%$ de la producción primaria total anual en sistemas de surgencia (Eppley \& Peterson 1979). Por otro lado, los flujos verticales de la fracción orgánica son menores en las regiones oceánicas sin surgencia correspondiendo al $14 \%$ de la producción primaria total. El restante de $86 \%$ de la producción primaria total corresponde a la producción primaria regenerada, sustentada por el reciclaje interno de elementos biogénicos en la columna de agua (Eppley \& Peterson 1979). Por lo tanto bajas tasas de sedimentación indican la predominancia de una producción primaria regenerada, y altas tasas la predominancia de la producción primaria nueva. Entretanto, en sistemas costeros esta relación es controlada por el aporte de fuentes adicionales. En aguas costeras los aportes del material biogénico y litogénico de origen terrestre sustentan una parte de la producción primaria nueva y alteran la señal de la sedimentación primaria de las aguas superficiales (Sanford 1992).

En este estudio las trampas de sedimentación fueron posicionadas en $30 \mathrm{~m}$ de profundidad incorporando la zona fótica y la termoclina/oxiclina, y en $80 \mathrm{~m}$ de profundidad zona afótica subóxica y a $20 \mathrm{~m}$

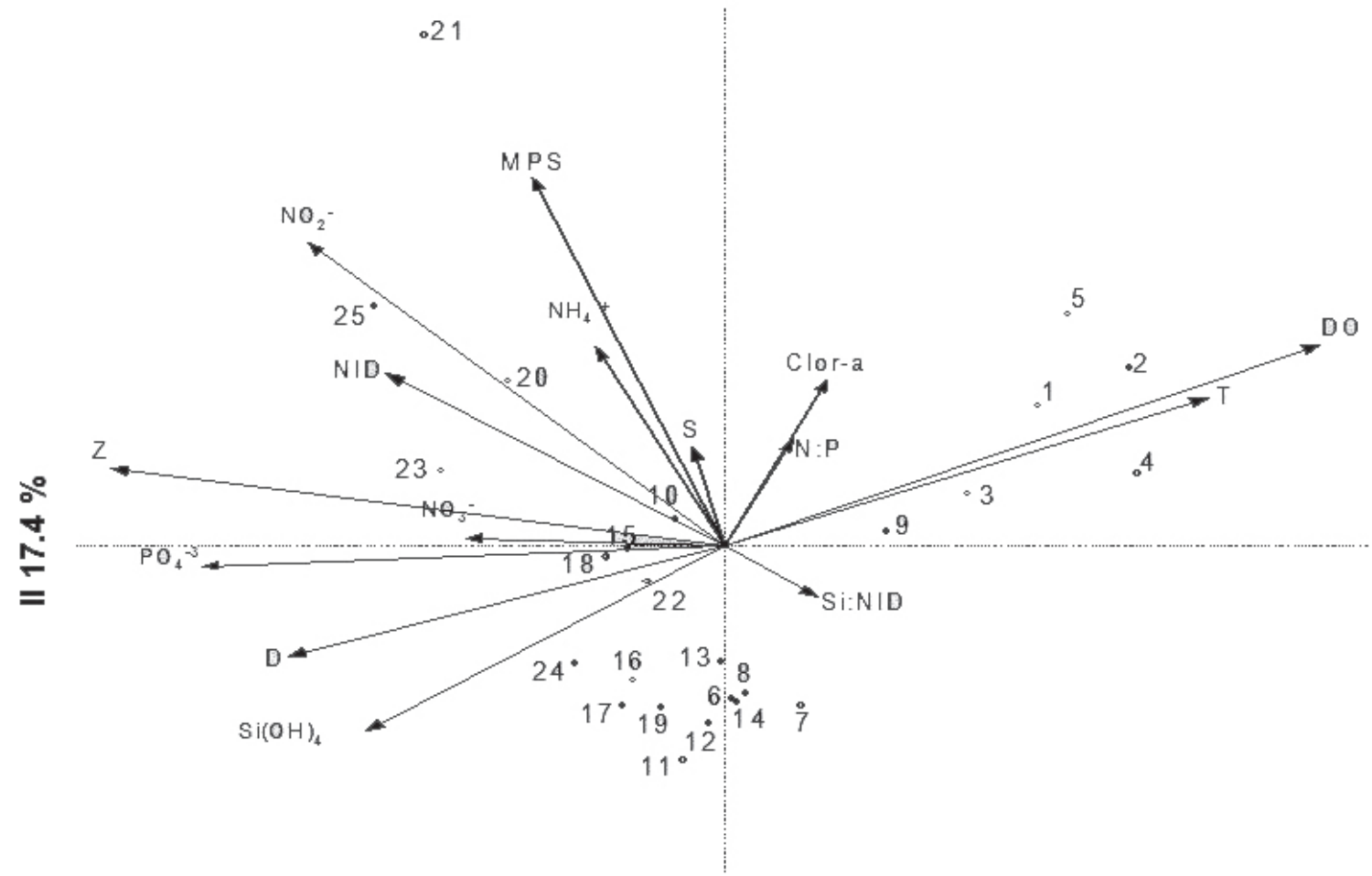

I $52.1 \%$

Fig. 6: Representación gráfica de los dos primeros componentes principales del análisis de la ACP, interpretando la matriz que contiene los parámetros físico-químicos y todas las muestras obtenidas en la estación fija de muestreo en la BM. $(\mathrm{T}=$ temperatura, $Z$ = profundidad, $\mathrm{D}=$ densidad, $\mathrm{MPS}=$ material partículado en suspensión, Clo-a = clorofila-a, Si:NID = razón entre ácido silícico y nitrógeno inorgánico disuelto y 1-25 = puntos de muestreo).

Graphic representation of both the two major components of the ACP analysis, interpreting the matrix that contains the physico-chemical parameters and all the samples obtained at the fixed station in BM. ( $\mathrm{T}=$ temperature. $Z=\mathrm{depth}, \mathrm{D}=$ density, MPS $=$ particulate suspended matter, Clo-a $=$ chlorophyll-a, Si:DIN $=$ ratio between silicic acid and dissolved inorganic nitrogen and numbers1-25 = sampling points). 
encima del fondo. Las variaciones quincenales de los flujos totales del material particulado colectados con las trampas instaladas en la BM (Tabla 1), sugieren que la sedimentación del material de la columna de agua está gobernada por diferentes procesos que dependen de la intensidad y duración de la surgencia, cuyo aporte de nutrientes incrementa la producción primaria nueva, y su alternancia con los periodos más prolongados de estratificación, que se desarrollan a lo largo del tiempo en sistemas caracterizados por la producción primaria regenerada. Los valores de los flujos verticales quincenales de las partículas durante el período de estudio, fueron relativamente bajos en comparación a otros sistemas de surgencia de la costa de Chile y Perú (Farías et al. 1994). Las tasas de sedimentación de los sólidos suspendidos totales (SST) de las trampas de 30 y $80 \mathrm{~m}$ alcanzaron valores medios de $1.1 \pm 0.4$ y $5.7 \pm$ $10.7 \mathrm{~g} \mathrm{~m}^{2} \mathrm{dia}^{-1}$, respectivamente. Estos valores coinciden con los periodos de mayor estratificación, que fueron antecedidos por períodos de surgencia. Las trampas de sedimentación en 30 y $80 \mathrm{~m}$ acompañaron muy bien las señales registradas en la columna de agua, con periodos de baja biomasa fitoplanctónica y altos flujos de sedimentación. Entretanto, las tasas de sedimentación registradas en las trampas posicionadas en $80 \mathrm{~m}$ de profundidad se mantuvieron dentro de un rango similar a las tasas de sedimentación reportadas por Vargas (2002) en testigos de sedimento recolectados en esta bahía. Estos autores indican una tasa líquida de acumulación de partículas, medidas con el exceso de ${ }^{210} \mathrm{~Pb}$, de $0.034 \mathrm{~g} \mathrm{~cm}^{2}$ año ${ }^{-1}$, lo que es equivalente a una tasa media de 8.7 $\mathrm{g} \mathrm{m}^{2}$ día $^{-1}$.

La razón C:N en el rango de 7:1 a 11:1 encontrada en el detrito orgánico colectado por las trampas de sedimento, indica la presencia de material todavía relativamente rico en nitrógeno semejante al fitoplancton intacto mezclado con una fracción del material detrítico nuevo. El detrito orgánico es proveniente de la producción primaria en la columna de agua, siendo removido por la sedimentación en forma de células intactas, pellets fecales y partículas detríticas amorfas. En las trampas de sedimentación se observó un patrón de empobrecimiento de nitrógeno en función de la profundidad, comparados con los valores registrados en sedimentos recientes debajo de las trampas (datos no publicados). El proceso de desnitrificación puede haber ocasionado esta discrepancia entre los valores de las trampas y el sedimento.

La relación entre las condiciones hidrodinámicas y la sedimentación de partículas biogénicas observada en este trabajo sugieren que el aumento del flujo de estas partículas estaría asociado a pulsos rápidos de surgencia y a la producción nueva generada por la entrada de nutrientes a la bahía. Esto se vería favorecido por una producción regenerada lenta, debido a que, bajo este escenario, la remineralización de la materia orgánica es menos eficiente. Esto corrobora el modelo conceptual propuesto por Valdés et al. (2004) para el interior de la BM. Altas tasas de producción primaria provocan el aumento de aglomeración y del peso específico de partículas orgánicas, que permite un tránsito más rápido de las partículas en la columna de agua y menor degradación del material en la columna de agua. Cuando hay una productividad baja, el proceso de floculación de partículas orgánicas es menos efectivo y la velocidad de decantación es más lenta. Como resultado de esto, el tiempo de residencia de las partículas en la columna de agua aumenta y la degradación de la materia orgánica es más eficiente. Los resultados sugieren que la sedimentación de partículas biogénicas reviste especial complejidad debido a la influencia de los pulsos de surgencia que ocurren periódicamente en la bahía de Mejillones del Sur.

\section{AGRADECIMIENTOS}

Este estudio se realizó dentro del Projecto JEAI (Jóvenes Equipos Asociados al IRD) «Laboratorio de Sedimentología y Paleoambientes» (LASPAL). El autor agradece la beca de estudio otorgada por la CAPES (Coordenação de Aperfeiçoamento de Pessoal de Nível Superior). Nuestros sinceros agradecimientos a la Universidad de Antofagasta. B. Knoppers es cientista senior del CNPq (Conselho Nacional para o Desenvolvimento Científico e Tecnológico, Beca Proc. No.306157/2007-3), Brasil, y al Dr. Humberto González por una revisión crítica de este manuscrito. Se agradecen también los comentarios de dos revisores anónimos y las sugerencias editoriales que ayudaron a mejorar el presente trabajo. 


\section{LITERATURA CITADA}

AHUMADA R (2002) Concepción bay a tectonic embayment-on the southeasthern Pacific coastline: A case study. En: Castilla JC \& JL Largier (eds) The oceanography and ecology of the nearshore and bays in Chile: 67-93. Pontificia Univesidad Católica de Chile, Santiago, Chile.

CAÑÓN J \& E MORALES (1985) Geografía del mar chileno. Instituto Geográfico Militar. Santiago, Chile.

ESCRIBANO R, V MARÍN, P HIDALGO \& G OLIVARES (2002) Physical-biological interactions in the nearshore zone of the northern Humboldt current system. En: Castilla JC \& JL Largier (eds) The oecanography and ecology of the nearshore and bays in Chile: 145175. Pontificia Univesidad Católica de Chile, Santiago, Chile.

ESCRIBANO R, SA ROSALES \& JL BLANCO (2004) Understanding upwelling circulation off Antofagasta (northern Chile): A threedimensional numerical-modeling approach. Continental Shelf Research 24: 37-53.

EPPLEY RW \& BJ PETERSON (1979) Particulate organic matter flux and planktonic new production in the deep ocean. Nature 80: 677679.

FARÍAS L, M SALAMANCA \& L CHUECAS (1994) Variaciones estacionales del flujo de partículas y contenido de materia orgánica a la interfase agua-sedimento en bahía Concepción, Chile Central. Ciencia y Tecnología del Mar 17: 15-31.

GONZÁLEZ H, G DANERI, D FIGUEROA, JL IRIARTE, N LEFĖVRE et al. (1998) Producción primaria y su destino en la trama trófica pelágica y océano profundo e intercambio océano atmósfera de $\mathrm{CO}_{2}$ en la zona norte de la Corriente de Humboldt ( $23^{\circ} \mathrm{S}$ ): Posibles efectos del evento El Niño 1997-1998. Revista Chilena de Historia Natural 71: 429-458.

GONZÁLEZ HE, VC ORTIZ \& M SOBARZO (2000) The role of faecal material in the particulate organic carbon flux in the northern Humboldt Current, Chile $\left(23^{\circ} \mathrm{S}\right)$, before and during the 1997-1998 El Niño. Journal of Plankton Research 22: 499-529.

GRASSHOFF K, M EHRHARDT \& M KREMLING (1983) Methods of seawater analysis. Springer Verlag, Basel, Switzerland.

HEUSSNER HS, A MONACO \& W FOWLER (1987) Characterization and vertical transpor of settling biogenic particles in the northwestern mediterranean. En: Degens ET, E Izdar \& S Honjo (eds) Particle flux in the ocean: 127-147. University Hamburg.

IRIARTE JL \& HE GONZÁLEZ (2004) Phytoplankton size structure during and after the 1997/98 El Niño in a coastal upwelling area of the northern Humboldt Current System. Marine Ecology Progress Series 269: 83-90.

ITTEKKOT V (1996) Particle flux in the ocean: Introduction. En: Ittekkot V, P Shafer, S Honyo \& PJ Depetris (eds) Particle flux in the ocean: 26. Scientific Committee On Problems of the Environment (SCOPE).

JAMES F \& C MCCULLOCH (1990) Multivariate analysis in ecology and systematics: Panacea or Pandora's box? Annual Review of Ecology Systematic 21: 129-166.
KNAUER GA, JH MARTIN \& KW BRULAND (1979) Fluxes of particulate carbon, nitrogen and phosphorus in the upper water column of the northeast Pacific. Deep-Sea Research 26: 97-108.

LETELIER J (1998) Estudio de la variabilidad de los eventos de surgencia, entre Arica $\left(18^{\circ} 29^{\prime} \mathrm{S}\right.$, $\left.70^{\circ} 19^{\prime} \mathrm{W}\right)$ y Tocopilla $\left(22^{\circ} 05^{\prime} \mathrm{S}, 70^{\circ} 11^{\prime} \mathrm{W}\right)$, observado en imágenes de satélite durante febrero y marzo de 1991 y 1992 . Tesis para optar al Título de Oceanógrafo, Pontificia Universidad Católica de Valparaíso, Valparaíso, Chile.

LIBES S (1992) An introduction to marine biogeochemestry. John Wiley \& Sons Press, New York, USA.

MARÍN V, L DELGADO \& R ESCRIBANO (2003) Upwelling shadows at Mejillones Bay (northern Chilean coast): A remote sensing in situ analysis. Investigaciones Marinas (Chile) 31: 4755.

MARÍN V, L RODRÍGUEZ, L VALLEJOS, J FUENTESECA \& E OYARCE (1993) Efectos de la surgencia costera sobre la productividad primaria primaveral de la bahía de Mejillones del Sur (Antofagasta, Chile). Revista Chilena de Historia Natural 66: 479-491.

MARÍN V \& G OLIVARES (1999) Estacionalidad de la productividad primaria en bahía de Mejillones del sur (Chile): Una aproximación procesofuncional. Revista Chilena de Historia Natural 72: 629-641.

PANTOJA S (2006) Biogeochemistry of the OMZ of Chile. Gayana 70: 62-67.

PAVES H \& HE GONZÁLEZ (2008) Carbon fluxes within the pelagic food web in the coastal area off Antofagasta ( $23^{\circ} \mathrm{S}$ ), Chile: The significance of the microbial versus classical food webs. Ecological Modelling 212: 218-232.

PIZARRO O, S HORMAZÁBAL, A GONZÁLEZ \& E YÁNEZ (1994) Variabilidad del viento, nivel del mar y temperatura en la costa norte de Chile. Investigaciones Marinas (Chile) 22: 83-101.

REDFIELD AC, BH KETCHUM \& FA RICHARDS (1963) The influence of organism on the composition of sea waters. En: Hill MN (ed) The sea: 26-77. John Wiley and Sons, New York, USA.

SANFORD L (1992) New sedimentation, resuspension and burial. Limnology Oceanography 37: 11641178.

STRICKLAND JDH \& TR PARSONS (1977) A practical handbook of seawater analysis. Second edition. Bulletin of Fisheries and Research. Ottawa, Canada.

SOKAL R \& F ROHLF (1981) Biometry-The principles and practice of statistics in biological research. Segunda edición. W.H. Freman, San Francisco.

TABACHNICK B \& L FIDELL (1989) Using multivariate statistics. Harper Collins Publishers, New York.

VALDES J, L ORTLIEB \& A SIFEDDINE (2003) Variaciones del sistema de surgencia de punta Angamos $\left(23^{\circ}\right)$ y la zona de mínimo oxígeno durante el pasado reciente. Una aproximación desde el registro sedimentario de la bahía de Mejillones del Sur. Revista Chilena de Historia Natural 76: 347-362.

VALDES J, A SIFEDDINE, E LALLIER-VERGES \& L ORTLIEB (2004) Petrographic and geochemical study of organic matter in surficial laminated sediments from an upwelling system (Mejillones 
del sur Bay, Northern Chile). Organic Geochemistry 35: 881-894.

VARGAS G (2002) Interactions oceans-atmosphere au cours des derniers siecle sur la côte Desert d'Atacama: Analyse multi-proxies des sediments lamines de Baie de Mehillones $\left(23^{\circ} \mathrm{S}\right)$. Tesis Doctoral, Departament de Geologie et
Oceanographie, Université de Bordeaux I, Bordeaux, France.

VARGAS CA \& HE GONZÁLEZ (2004) Plankton community structure and carbon cycling in a coastal upwelling system. II. Microheterotrophic pathway. Aquatic Microbial Ecology 34: 165-180.

Editor Asociado: Humberto E. González

Recibido el 18 de abril de 2008; aceptado el 1 de junio de 2010 